Article

\title{
Virtual Network Provisioning over Mixed-Fixed/Flexible-Grid Optical Infrastructures
}

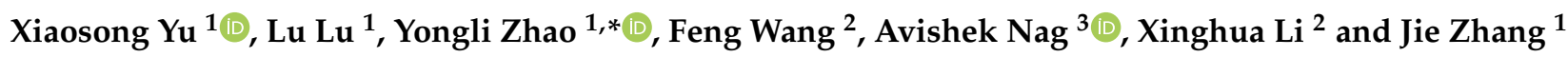 \\ 1 State Key Laboratory of Information Photonics and Optical Communications (IPOC), Beijing University \\ of Posts and Telecommunications (BUPT), Beijing 100876, China; xiaosongyu@bupt.edu.cn (X.Y.); \\ lululu@bupt.edu.cn (L.L.); lgr24@bupt.edu.cn (J.Z.) \\ 2 State Grid Electric Power Technical Research Institute, Yinchuan 750001, China; \\ wangfeng@dky.nx.sgcc.com.cn (F.W.); lixinghua@dky.nx.sgcc.com.cn (X.L.) \\ 3 School of Electrical and Electronic Engineering, University College Dublin, Dublin 4 D04 V1W8, Ireland; \\ avishek.nag@ucd.ie \\ * Correspondence: yonglizhao@bupt.edu.cn
}

Citation: Yu, X.; Lu, L.; Zhao, Y.; Wang, F.; Nag, A.; Li, X.; Zhang, J. Virtual Network Provisioning over Mixed-Fixed/Flexible-Grid Optical Infrastructures. Electronics 2021, 10, 2067. https://doi.org/10.3390/ electronics10172067

Academic Editor: Christos J. Bouras

Received: 10 July 2021

Accepted: 24 August 2021

Published: 26 August 2021

Publisher's Note: MDPI stays neutral with regard to jurisdictional claims in published maps and institutional affiliations.

Copyright: (c) 2021 by the authors. Licensee MDPI, Basel, Switzerland. This article is an open access article distributed under the terms and conditions of the Creative Commons Attribution (CC BY) license (https:// creativecommons.org/licenses/by/ $4.0 /)$.

\begin{abstract}
With the emergence of cloud services based on data centers, demands for bandwidthintensive applications have increased dramatically, and application services have transferred to a more diversified direction. Management as well as capacity of the backbone network needs further development to catch up with rapidly evolved application demands. Optical network virtualization can facilitate the sharing of physical infrastructure among multiple network applications. Virtual Network Embedding (VNE), the main implementation of network virtualization, determines how to map a virtual network request onto physical substrate. To expand the network capacity, flexiblegrid elastic optical networks have been considered as a promising supporting technology for the future infrastructure of the next-generation Internet. However, due to the expense of key enabling equipment for flexible grid optical networks, the brown-field migration from a fixed grid to a flexible grid gave birth to the co-existing fixed/flexible grid. Based on the co-existing fixed/flexible grid optical networks, we investigate the problem of Virtual Optical Network (VON) provisioning, and present a flexible-grid-aware virtual network embedding algorithm to map the virtual networks onto the substrate network. In addition, the performance of the algorithm was evaluated under four different network scenarios. Simulation results show that the proposed algorithm can achieve better performance in all four scenarios.
\end{abstract}

Keywords: Virtual Optical Network (VON); flexible grid; WDM; Virtual Network Embedding (VNE); mixed-grid

\section{Introduction}

With large-scale deployment of data centers and continuing proliferation of cloudbased applications, traffic has grown dramatically and is imposing a huge demand on network bandwidth. To handle such fast applications deployment and dynamic data traffic, network virtualization was proposed as a promising solution, which can lead to reduced cost and improved networking hardware utilization [1-4]. In addition, network virtualization allows network service providers to flexibly provide new and innovative services over substrate network infrastructure [5-8]. Network virtualization is widely used in data center networks, and it is also an important technology of 5 G network slicing $[9,10]$. Optical network virtualization enables network operators to compose and operate multiple independent and application-specific Virtual Optical Networks (VONs) simultaneously sharing a common physical optical network infrastructure. A typical VON is composed of several virtual nodes interconnected by virtual links. Each virtual node requires a certain amount of computing resources from the substrate nodes, while each virtual link requires a spectrum path consisting of one or multiple substrate fiber links between two substrate 
nodes associated with two virtual nodes [2]. The process of mapping virtual nodes/links to substrate nodes/links is called Virtual Network Embedding (VNE).

In the literature, researchers have studied the VNE problems in optical networks from different perspectives [11,12]. VNE was extensively studied for layer-2/3 electrical networks [5]. Fixed-grid dense wavelength division multiplexing technology is typically used by the existing optical layer [13]. The $50 \mathrm{GHz}$ International Telecommunication Union (ITU) wavelength grid divides the C-band optical spectrum into fixed $50 \mathrm{GHz}$ spectrum slots, and 40-80 such wavelengths can be carried on a fiber pair. Some work was performed for VNE in Fixed-grid optical networks [1,14]. Through the network design with flexible virtual node mapping, efficient utilization of resources can be realized [1]. In [14], the proposed PLI-aware VON composition methods can dynamically compose multiple coexisting and cost-effective VONs with guaranteed transmission quality. Although Dense Wavelength Division Multiplexing (DWDM) increases fiber utilization, constrains remain for further expanding network capacity. For instance, coarse-grained spectrum allocation in DWDM leads to wasted capacity, and fixed-grid DWDM is not flexible enough to meet the growing need for supporting data-rates beyond $100 \mathrm{Gbps}$ [15]. As one of the physical substrate network technologies, flexible grid networks [16] are more spectrally efficient than traditional wavelength division multiplexing networks. It evolves the traditional ITU grid towards higher flexibility with fine-grained spectrum slots, and it can significantly increase the overall network capacity by using on-demand spectrum assignment and advanced adaptive modulation formats.

In recent years, the VNE problem in flexible grid optical networks has received much attention from the research communities [2,15,17-24]. S. Ding et al. proposed a trading scheme to trade spectra between VONs embedded in an Elastic Optical Network (EON) for efficient spectrum utilization and better client quality of service [18]. To avoid the mismatch between the assigned-capacity and the actual carried traffic demand, a spectrum trading scheme to trade spectrum resources between VONs in EON was proposed [19]. From the perspective of distance-and-modulation-adaptive Routing and Spectrum Allocation (RSA), [20] addressed the $\mathrm{VN}$ embedding problem with the goal of maximizing the number of embedded VNs over software-defined optical networks for the first time. In [21], the authors solved the VNE problem with geographical constraints in the context of flexi-grid optical networks where modulation modes can be optimally selected. A fragmentationaware Virtual Optical Network Embedding (VONE) algorithm is designed by coordinating node and link mapping during VONE to reduce spectrum fragmentation and improve resource unitization [22]. Nevertheless, since the key enabling equipment for flexible grid optical networks (e.g., flexible wavelength-selective switches which support non-uniform channels) are expensive, it may not be economically viable to make a one-time upgrade from fixed-grid to flexible-grid technology on the entire network. In other words, before the current optical transport network capacity is exhausted, the current fixed-grid network could be maximally kept operational during the migration to preserve the already-made investment. As a result, brownfield flexible-grid deployment on top of the existing fixedgrid network is highly likely to happen $[25,26]$.

There are some works that addressed the RSA problem [27-30] or considered costs [31-34] in co-existing fixed/flexible grid networks. In [27], the static RSA in coexisting fixed/flex grid optical networks is considered and Integer Linear Programming (ILP) formulations are presented to minimize the utilized spectrum. In [24], the problem of gradual migration from fixed grid to flex grid is addressed. However, the state-of-the-art in co-existing fixed/flexible grid networks rarely takes the VON provisioning issues into consideration. Our previous work addressed the VON provisioning issues in co-existing fixed/flex grid optical networks for the first time [35]. In this paper, we extend the above work and evaluate performance of the proposed flexible-grid aware VNE algorithm. The remainder of this paper is organized as follows. The problem statement of VON provisioning over fixed/flexible grid optical networks is illustrated in Section 2. Section 3 describes the network model first, and then introduces the heuristic VON provisioning algorithm based 
on the basic network model. Simulation performances in terms of blocking probability and link utilization are evaluated in Section 4. Finally, Section 5 summarizes this paper.

\section{Problem Statement}

The physical optical network infrastructure is simplified to substrate nodes and substrate links, while the virtual optical network is composed of virtual nodes and virtual links. When VON requests come one after another, a VON provisioning procedure is needed to map the virtual nodes/links of each VON request onto the substrate nodes/links. In co-existing fixed/flexible grid optical networks, there are two different kinds of nodes: (1) fixed-grid nodes, which follow traditional rigid ITU-T-defined central frequencies and spectrum grids (e.g., $50 \mathrm{GHz}$ ); and (2) flexible-grid nodes which do not need to strictly follow the fixed frequency grid and can switch spectral bandwidth as an integer multiple of finer-granularity slices (e.g., $6.25 \mathrm{GHz}$ ). To achieve more efficient utilization of the network spectrum resources, flexible-grid nodes should be selected with a high-priority in the process of VNE, since more spectrally-efficient lightpaths can be setup. Figure 1 shows an illustrative example. Suppose there is a VON request $R_{1}$. The computing requirements of virtual node $\mathrm{a}, \mathrm{b}, \mathrm{c}$ in $R_{1}$ are 5 units, and the bandwidth requirements of virtual link ab, bc are $200 \mathrm{~Gb} / \mathrm{s}$. The substrate optical network consists of 8 fixed-grid nodes and 6 flexiblegrid nodes, and their computing capacities are 300 units. Due to geographic constraints, the candidate mapping nodes for $a, b, c$ are $\{1,2,3,4\},\{5,6,7\}$, and $\{10,12,13,14\}$, respectively. Obviously, the consumption of substrate optical network resources will be minimized if the node mapping for $R_{1}$ is $\{\mathrm{a} \rightarrow 3, \mathrm{~b} \rightarrow 6, \mathrm{c} \rightarrow 13\}$, since two flexible-grid super-channels can be set up (from node 3 to node 6 for link ab, and from node 6 to node 13 for link bc).

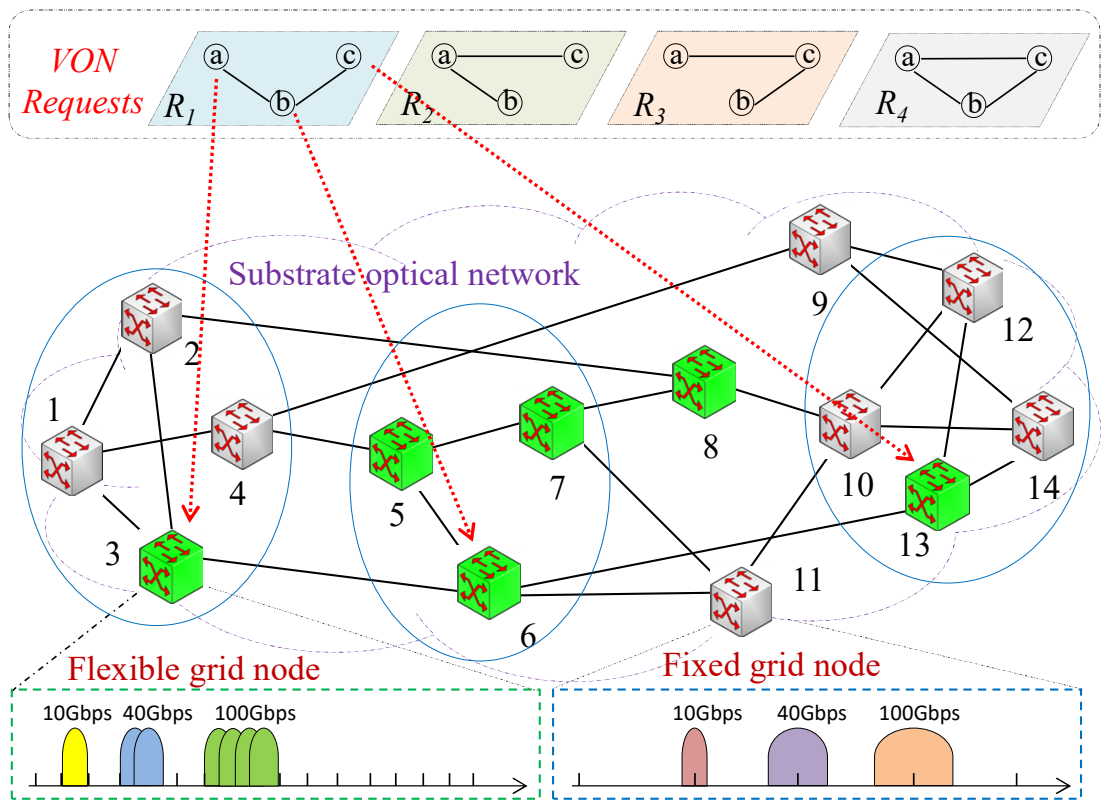

Figure 1. VON provisioning over fixed/flexible grid optical networks.

The co-existing of fixed-grid nodes and flexible-grid nodes also leads to two kinds of spectrum, which constrains joining into the RSA problem. Spectrum assignment cases are shown in Figure 2. Here we assume that the fixed-grid node spectral bandwidth is $50 \mathrm{GHz}$ and the flexible-grid node spectral bandwidth is $12.5 \mathrm{GHz}$. The spectrum is shared as the common resources, and four cases will exist for VON requests due to the routing with different type of passed nodes in mixed-fixed/flex grid optical networks (Figure 2). We can see that when the source node is a fixed-grid node, the spectrum resources for each link on this path can only be allocated according to the fixed-grid granularity slice ( $50 \mathrm{GHz})$. The reason is the switching granularity of the fixed-grid node cannot be smaller than $50 \mathrm{GHz}$ [20], and the next frequency spectrum will only be carried at a coarser granularity, 
regardless of whether it is a fixed-grid or flexible-grid node. If the source node is a flexiblegrid node, it can occupy a spectrum frequency closer to the size of the service. However, if the path needs to pass through one or more fixed-grid node(s), the frequency still needs to be converted to $50 \mathrm{GHz}$ at the fixed-grid node, as shown in Figure 2c.

(a)

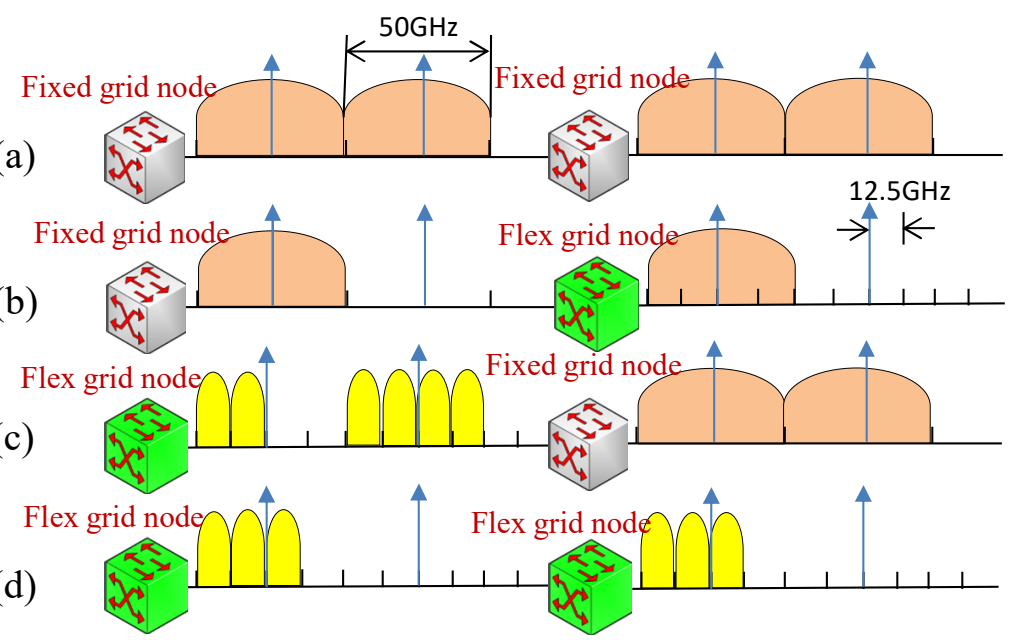

Figure 2. Spectrum assignment cases in mixed-fixed/flexible-grid optical infrastructures: (a) origin from fixed grid node and pass fixed grid node; (b) origin from fixed grid node and pass flex grid node; (c) origin from flex grid node and pass fixed grid node; (d) origin from flex grid node and pass flex grid node.

\section{Network Model and VON Provisioning Algorithm}

Based on the idea described in Section 2, we model the network in Section 3.1, and then a flexible-grid aware VNE algorithm is proposed in Section 3.2. Also, we give a brief description for a flexible-grid unaware VNE algorithm, as a comparison.

\subsection{Network Model}

Several parameter definitions are listed in Table 1. Suppose the substrate network is modeled as an undirected weighted graph $G^{S}=\left\{V^{S}, E^{S}\right\}$, where $V^{S}=\left\{v_{i}^{S}, i=1,2, \ldots N\right\}$ denotes the set of substrate nodes ( $N$ is the total number of nodes, including fixed-grid and flexible-grid), and $E^{S}=\left\{e_{j}^{s}, j=1,2, \ldots L\right\}$ denotes the set of substrate fiber links ( $L$ is the total number of links). The computing capacity of a substrate node $v_{i}^{s}$ is expressed as $C_{c}\left(v_{i}^{s}\right)$, while the bandwidth capacity of a substrate fiber link $e_{j}^{s}$ is denoted as $C_{b}\left(e_{j}^{s}\right)$. Similarly, the VON requests are modeled as $G^{V}=\left\{G_{m}^{V}, m=1,2, \ldots M\right\}$, where $M$ is the total number of VON requests. More specifically, $G_{m}^{V}=\left\{V_{m}^{V}, E_{m}^{V}\right\}$, where $V_{m}^{V}=\left\{v_{m_{x}}^{v}, x=1,2, \ldots n\right\}$ denotes the set of virtual nodes for $\mathrm{VON}$ request $G_{m}^{V}$ ( $n$ is the total number of virtual nodes), and $E_{m}^{V}=\left\{e_{m_{y}}^{v}, y=1,2, \ldots l\right\}$ denotes the set of virtual links for VON request $G_{m}^{V}(l$ is the total number of virtual links). The computing requirement of a virtual node $v_{m_{x}}^{v}$ is expressed as $R_{c}\left(v_{m_{x}}^{v}\right)$, while the bandwidth requirement of a virtual link $e_{m_{y}}^{v}$ is denoted as $R_{b}\left(e_{m_{y}}^{v}\right)$. The virtual network provisioning problem can be defined as: given the substrate network $G^{S}=\left\{V^{S}, E^{S}\right\}$ and any VON request $G_{m}^{V}=\left\{V_{m}^{V}, E_{m}^{V}\right\}$, we need to find the mapping of VON nodes and links to the substrate nodes and links (i.e., $M_{N}\left(V_{m}^{V}\right)$ and $M_{L}\left(E_{m}^{V}\right)$ ) while satisfying the requirements: (1) a virtual node $v_{m_{x}}^{v}$ must be mapped to only one substrate node $v_{i}^{s}$ such that $R_{c}\left(v_{m_{x}}^{v}\right)<C_{\mathcal{c}}\left(v_{i}^{s}\right)$; and (2) a virtual link $e_{m_{y}}^{v}$ must be mapped to a spectrum path including one/several substrate $\operatorname{link}(\mathrm{s})$, i.e., $e_{j}^{s}(j=1, \ldots)$, such that $R_{b}\left(e_{m_{y}}^{v}\right)<C_{b}\left(e_{j}^{s}\right)$ for each substrate link $e_{j}^{s}$. 
Table 1. Parameter definitions.

\begin{tabular}{|c|c|}
\hline Parameters & Definitions \\
\hline$G^{S}=\left\{V^{S}, E^{S}\right\}$ & the substrate network \\
\hline$V^{S}=\left\{v_{i}^{S}, i=1,2, \ldots N\right\}$ & the set of substrate nodes ( $N$ is the total number of nodes) \\
\hline$E^{S}=\left\{e_{j}^{s}, j=1,2, \ldots L\right\}$ & the set of substrate fiber links ( $L$ is the total number of links) \\
\hline$C_{c}\left(v_{i}^{s}\right)$ & the computing capacity of $v_{i}^{s}$ \\
\hline$C_{b}\left(e_{j}^{s}\right)$ & the bandwidth capacity of $e_{j}^{s}$ \\
\hline$G_{m}^{V}=\left\{V_{m}^{V}, E_{m}^{V}\right\}$ & the VON requests \\
\hline$V_{m}^{V}=\left\{v_{m_{x}}^{v}, x=1,2, \ldots n\right\}$ & $\begin{array}{l}\text { the set of virtual nodes for VON request } G_{m}^{V}(n \text { is the total } \\
\text { number of virtual nodes })\end{array}$ \\
\hline 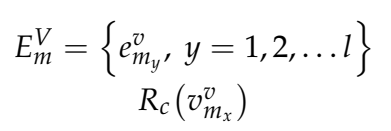 & $\begin{array}{c}\text { the set of virtual links for VON request } G_{m}^{V}(l \text { is the total } \\
\text { number of virtual links }) \\
\text { the computing requirement of } v_{m_{x}}^{v}\end{array}$ \\
\hline$R_{b}\left(e_{m_{y}}^{v}\right)$ & the bandwidth requirement of $e_{m_{y}}^{v}$ \\
\hline$M_{N}\left(V_{m}^{V}\right)$ & the mapping of VON nodes to the substrate nodes \\
\hline$M_{L}\left(E_{m}^{V}\right)$ & the mapping of VON links to the substrate links \\
\hline
\end{tabular}

\subsection{VON Provisioning Algorithm}

The VNE problem is considered as an NP-hard problem; it refers to the process of allocating physical resources to virtual nodes and virtual links [4]. Thus, the problem of VON Provisioning can be extremely hard; the optimal mapping is affected by many factors such as network topology, routing and wavelength/spectrum allocation algorithms, network traffic load, traffic distribution, etc. In such a situation, heuristics are and will stay useful for approaching the optimal mapping. In this section, we propose a heuristic algorithm called Flexible-grid Aware-Virtual Network Embedding Algorithm (FA-VNE). The detailed procedures are shown in Algorithm 1. The process of VON Provisioning mainly consists of two parts, virtual node mapping and virtual link mapping. First, we need to initialize the result set and sort the nodes in $V_{m}^{V}$ and $V^{S}$ for fixed/flexible-grid nodes based on $p\left(v_{m_{x}}^{v}\right)$ and $p\left(v_{i}^{s}\right)$ in descending order, respectively. Note that the node degree as well as its computing requirement (computing capacity) are considered when ordering the virtual (substrate) nodes in $V_{m}^{V}\left(V^{S}\right)$. Specifically, the value of $p\left(v_{m_{x}}^{v}\right)$ and $p\left(v_{i}^{s}\right)$ are computed as $p\left(v_{m_{x}}^{v}\right)=R_{c}\left(v_{m_{x}}^{v}\right) \times d\left(v_{m_{x}}^{v}\right)$ and $p\left(v_{i}^{s}\right)=C_{c}\left(v_{i}^{s}\right) \times d\left(v_{i}^{s}\right)$ respectively, where $d\left(v_{m_{x}}^{v}\right)$ and $d\left(v_{i}^{s}\right)$ are the nodal degree of $v_{m_{x}}^{v}$ and $v_{i}^{s}$. Here, the geographic constraints are not considered to simplify the problem.

As for the virtual node mapping, flexible nodes have finer granularity, which leads to more flexible spectrum allocation and less spectrum waste. Thus, the virtual nodes are set to be prone mapped onto flexible-grid nodes in the substrate network in order to make the best use of the flexible-grid technology, which is called Flexible-grid Aware. Traversing each $v_{i}^{S}$ in $V^{S}$ and $v_{m_{x}}^{v}$ in $V_{m}^{V}$ following a virtual node $v_{m_{x}}^{v}$ mapped to only one substrate node $v_{i}^{s}$, until each $v_{m_{x}}^{v}$ in $V_{m}^{V}$ maps to the most appropriate $v_{i}^{s}$. $V^{S-f l e x}$ is preferred versus $V^{S-f i x}$ in traversal. If neither $V^{S-f l e x}$ nor $V^{S-f i x}$ can be mapped to a VON request $G_{m}^{V}$, node mapping fails and this request is blocked. When the virtual node mapping succeed, link mapping can be executed then. A spectrum path $P^{S}\left(e_{m_{y}}^{v}\right)$ is chosen under the requirement that a virtual link $e_{m_{y}}^{v}$ must be mapped to a spectrum path including one/several substrate link(s). If the spectrum path from $M_{N}\left(v_{m_{x 1}}^{v}\right)$ to $M_{N}\left(v_{m_{x 2}}^{v}\right)$ cannot be found, block request $G_{m}^{V}$. Note in line 24, the RSA algorithm that satisfies the fixed/flexible-grid spectrum constraint is used for finding a path $P^{S}\left(e_{m_{y}}^{v}\right)$ in $G^{S}$, which could be found in $[25,27,28]$. 


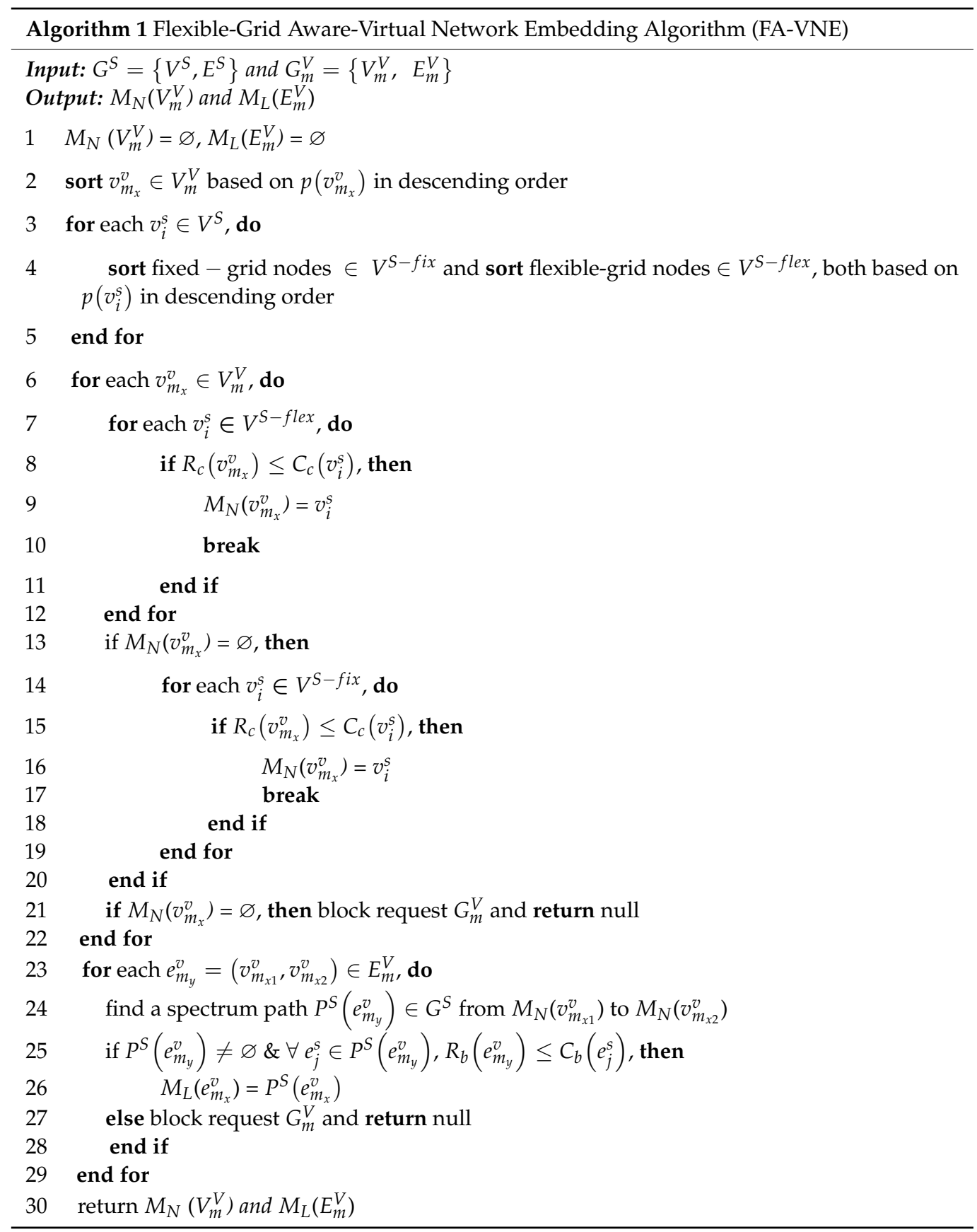

The computational complexity related to this algorithm is bounded by $O\left(\left|V^{S}\right|+\left|V_{m}^{V} \times V^{S}\right|+\left|E_{m}^{V}\right|\right)$. Lines 1 to 5 require $\left|V^{S}\right|$ operations. Lines 7 to 12 require at most $\left|V^{S-\text { flex }}\right|$ operations. Similarly, lines 13 to 20 require at most $\left|V^{S-f i x}\right|$ operations. Lines 6 to 22 require at most $\left|V_{m}^{V}\right| \times\left(\left|V^{S-f l e x}\right|+\left|V^{S-f i x}\right|\right)=\left|V_{m}^{V} \times V^{S}\right|$ operations. Lines 23 to 29 require $\left|E_{m}^{V}\right|$ operations. $\left|V^{S}\right|+\left|V_{m}^{V} \times V^{S}\right|+\left|E_{m}^{V}\right|$ corresponds to the worst-case complexity of finding the mapping.

In the performance Evaluation part, the Flexible-grid Unaware-Virtual Network Embedding Algorithm (FU-VNE), which does not consider the flexible-grid nodes with higherpriority during VON provisioning, is compared as the baseline. The procedure of the $F U-V N E$ algorithm is shown in Algorithm 2. 


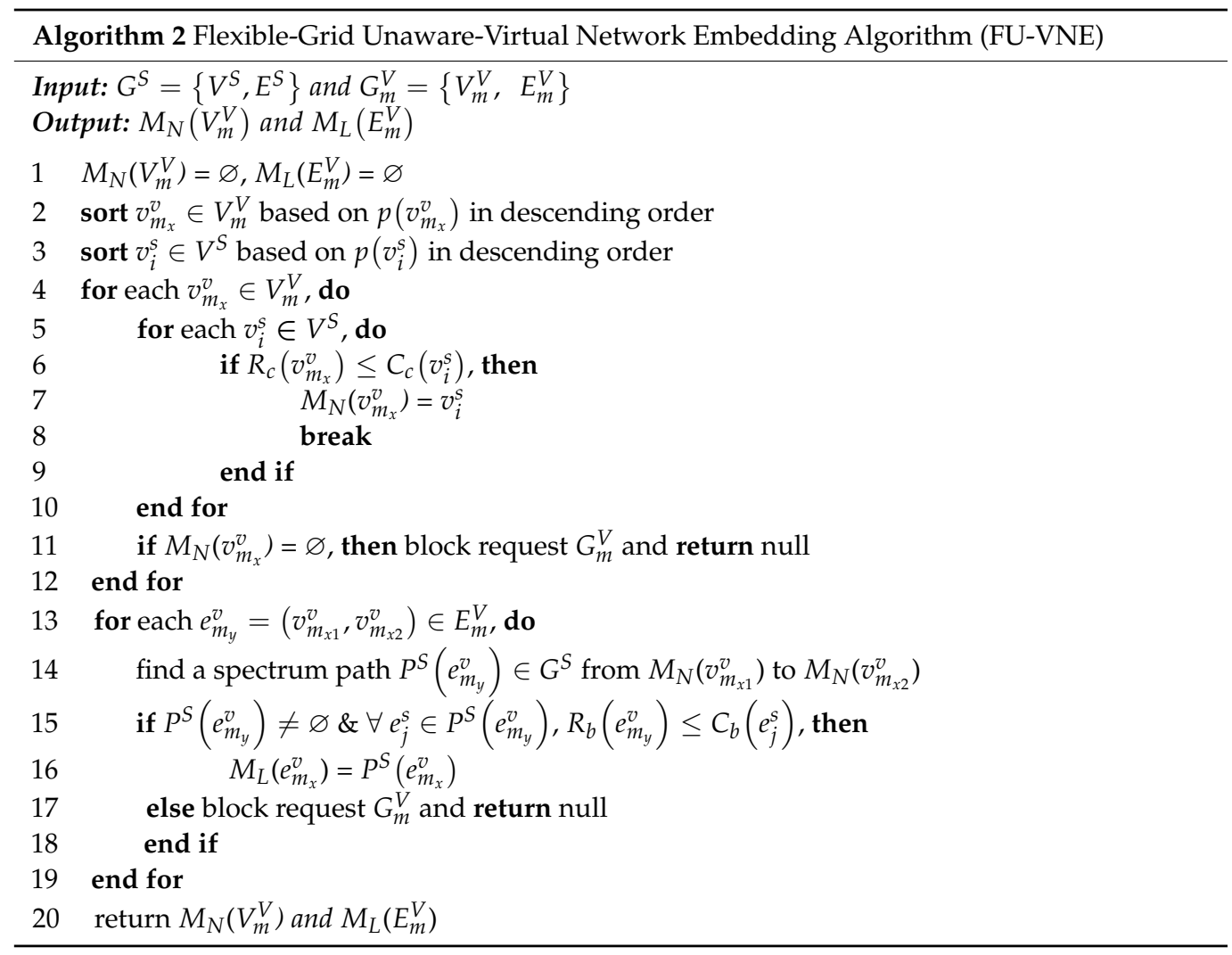

\section{Performance Evaluation}

We evaluate the performance of the proposed $F A-V N E$ algorithm in the 14-node NSFNET topology shown in Figure 3, where the computing capacity of a substrate node is set to 500 units, and the computing requirements of a virtual node are randomly chosen from $[1,3]$ units. The spectrum capacity in a substrate link is set to $8 \mathrm{THz}$ (evenly divided in each direction), and the bandwidth requirements of a virtual link are randomly chosen among [40, 100, 200, 400] Gb/s. For these demands, [50, 50, 100, 200] GHz spectrum are assumed to be required by fixed-grid nodes/links, while [25, 37.5, 75, 125] GHz spectrum are assumed to be required by flexible-grid nodes/links (the distance-adaptive modulation format is not considered here). Following Poisson arrivals, 50,000 VON requests are generated (the serving rate is fixed to 1 , so the traffic load is determined by the arriving rate). Each VON request is generated with $[3,5]$ virtual nodes, and the virtual links between virtual nodes are randomly created by the Erdos-Renvi model, with a probability $\alpha(\alpha$ is set to 0.5 and 0.7 in the simulation). The KSP routing algorithm is used to map virtual links with $K=3$. During simulation, all VON requests are served in a First-Come-First-Served (FCFS) manner. We consider four different Network Scenarios (NS) as shown in Table 2. In NS- $A$ and NS-B, low-bandwidth demands (e.g., $40 \mathrm{~Gb} / \mathrm{s}$ ) are predominant, while in NS-C and NS-D, high-bandwidth demands (e.g., $200 \mathrm{~Gb} / \mathrm{s}$ and $400 \mathrm{~Gb} / \mathrm{s}$ ) are predominant, compared with low-bandwidth ones. Additionally, there are 30\% flex-nodes in NS- $A$ and $N S-C$, and $60 \%$ flex-nodes in NS-B and NS-D. 


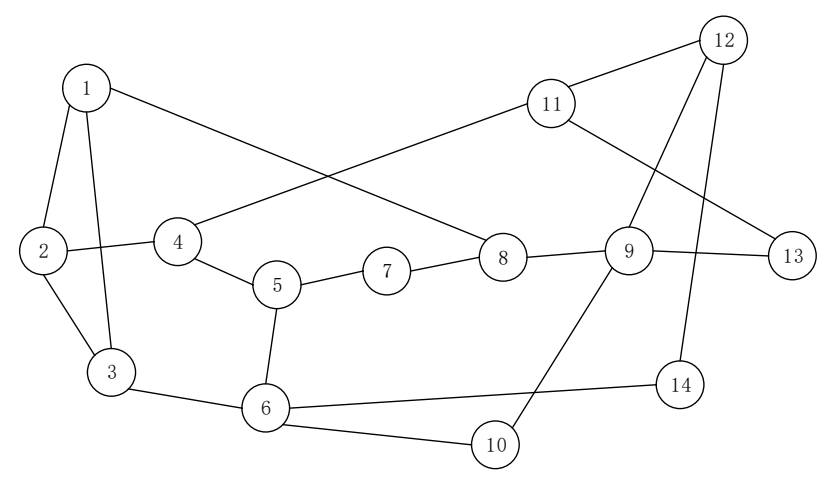

Figure 3. NSFNET topology used for simulation.

Table 2. Parameters in different Network Scenarios (NS).

\begin{tabular}{cccc}
\hline Parameters & Bandwidth (Gb/s) & Percentage & Flex-Grid Nodes \\
\hline \multirow{2}{*}{ Network Scenario A (NS-A) } & 40 & $50 \%$ & \\
& 100 & $30 \%$ & $30 \%$ \\
Network Scenario B (NS-B) & 200 & $15 \%$ & \\
& 400 & $5 \%$ & \\
& 40 & $50 \%$ & $60 \%$ \\
Network Scenario C (NS-C) & 200 & $30 \%$ & \\
& 400 & $15 \%$ & $30 \%$ \\
& 40 & $10 \%$ & \\
& 200 & $40 \%$ & \\
Network Scenario D (NS-D) & 400 & $30 \%$ & $60 \%$ \\
\hline
\end{tabular}

In the simulation, we use the FU-VNE algorithm as a baseline for comparison. Figure 4a-d shows the performance in terms of the Blocking Probability (BP) of $F A-V N E$ and $F U-V N E$ under different NSs. Each sub graph in Figure 4 takes blocking probability as the y-axis, which represents the blocking probability of the VON services in each NS. Taking traffic load as the $x$-axis, the values shown at each point are the expected values of the blocking probability of $5000 \mathrm{VON}$ Poisson services under the corresponding traffic load. The first bar and the third bar of each traffic load are the proposed FA-VNE algorithm with $\alpha$ values of 0.5 and 0.7 , while the second bar and the fourth bar are FU-VNE as a comparison algorithm, and $\alpha$ value is also set to be 0.5 and 0.7 , respectively.

The result in Figure 4a is obtained by simulating NS- $A$, where low-bandwidth demands (e.g., $40 \mathrm{~Gb} / \mathrm{s}$ ) dominate and contain fewer flexible-grid nodes (30\%). It can be seen from Figure $4 \mathrm{a}$ that blocking probabilities increase with the increase of traffic load for all cases. Additionally, considering a flexible-grid aware approach can reduce the blocking probabilities up to $52 \%$, which indicates that $F A-V N E$ greatly improves the network performance compared with $F U-V N E$. It is an interesting phenomenon that when the probability of virtual link generation is set to 0.5 ( $\alpha$ is 0.5 ) in the FA-VNE and FU-VNE algorithms, lower blocking probability can be achieved in both cases. Thus, higher probability of virtual link generation does not mean that the network performance improves. From the other subgraphs in Figure 4, we can obtain the same information as Figure 4a. Figure $4 \mathrm{~b}$ is obtained by simulating $N S-B$, where low-bandwidth demands (e.g., $40 \mathrm{~Gb} / \mathrm{s}$ ) dominate and contain more flexible-grid nodes (30\%). By comparing Figure $4 a$ and $b$, we find that when the percentage of flexible-grid node increases, $F A-V N E$ has a more prominent advantage in blocking probability than $F U-V N E$. The reason is that $F A-V N E$ is prone to select flexible-grid nodes in the process of node mapping for the incoming VON requests, 
and more flexible-grid based channels can be set up to save spectrum resources in the substrate optical network, which can contribute to reduce the VON blocking probability. The comparison of Figure 4c,d shows a similar trend, which were respectively obtained by simulating NS-C and NS-D. Also note that with the increase of high-bandwidth VON demands, $F A-V N E$ shows certain advantages by comparing Figure $4 \mathrm{a}$,c or Figure $4 \mathrm{~b}$,d. For example, when the traffic load is 880 Erlang, the blocking probability of $F A-V N E$ is $22.6 \%$ lower than FU-VNE when $\alpha$ is 0.5 and $16.5 \%$ lower than FU-VNE when $\alpha$ is 0.7 in Figure $4 \mathrm{~b}$, while in Figure $4 \mathrm{~d}$, the percentage is $27.5 \%$ and $23.4 \%$ respectively

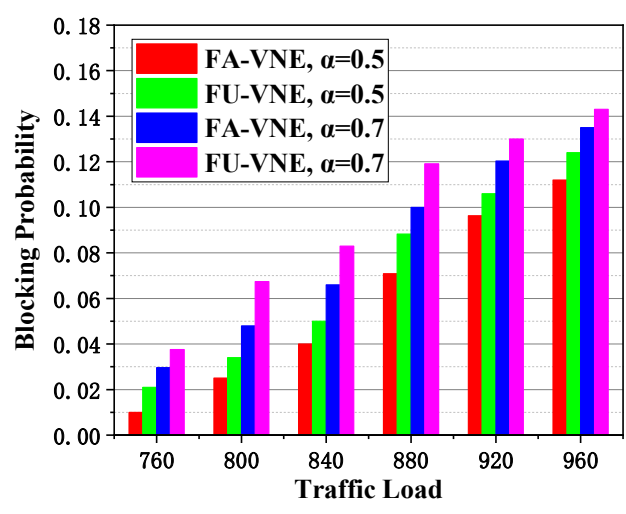

(a)

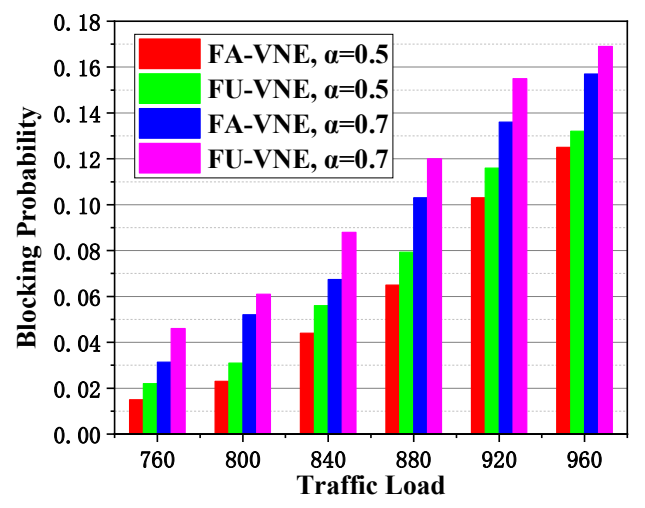

(c)

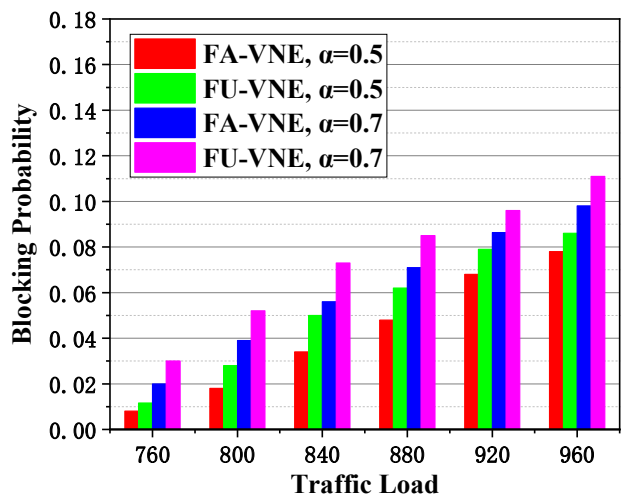

(b)

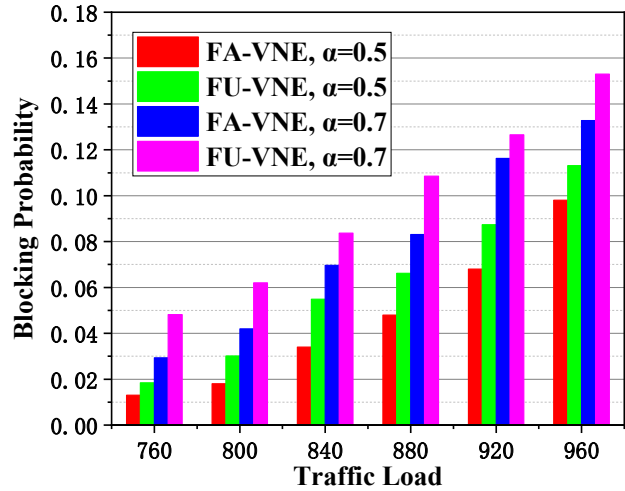

(d)

Figure 4. Simulation and results: (a) BP under NS-A; (b) BP under NS-B; (c) BP under NS-C; and (d) BP under NS-D.

The performance of $F A-V N E$ and that of $F U-V N E$, in terms of Link Utilization Ratio (LUR), are also evaluated as shown in Figure 5a-d for different NSs. The link utilization ratio is defined as the ratio of average amount of bandwidth used to the total bandwidth in the link. The first and third curves of each traffic load are the link utilization ratios of the proposed $F A-V N E$ algorithm with different $\alpha$ values, the second and fourth curves are the link utilization ratios of FU-VNE. The result in Figure 5a is obtained by simulating NS- $A$. From the figure we can see that the link utilization ratio increases with the increase in the traffic load for all cases, with FA-VNE performing $16 \%$ better than FU-VNE. The reason is $F A-V N E$ better identifies node/link mapping with less blocking probability. Additionally, higher link utilization is also achieved when $\alpha$ is 0.5 . Higher probability of virtual link generation failed to achieve excellent performance in terms of both blocking probability and link utilization ratio. From the other subgraphs in Figure 5, we can obtain the same information as Figure $5 \mathrm{a}$. Figure $5 \mathrm{~b}$ is obtained by simulating NS-B. The comparison between Figure $5 \mathrm{a}, \mathrm{b}$ or Figure $5 \mathrm{c}$, $\mathrm{d}$ shows when the network contains more flexible-grid nodes, the advantage is a little more obvious. For example, the highest improvement of $F A-V N E$ is $15 \%$, while it is $11 \%$ for FU-VNE. Also, the comparison between Figure 5a,b or Figure $5 c$,d manifests as high-bandwidth VON demands increase, as the advantage of FA-VNE becomes more obvious. 


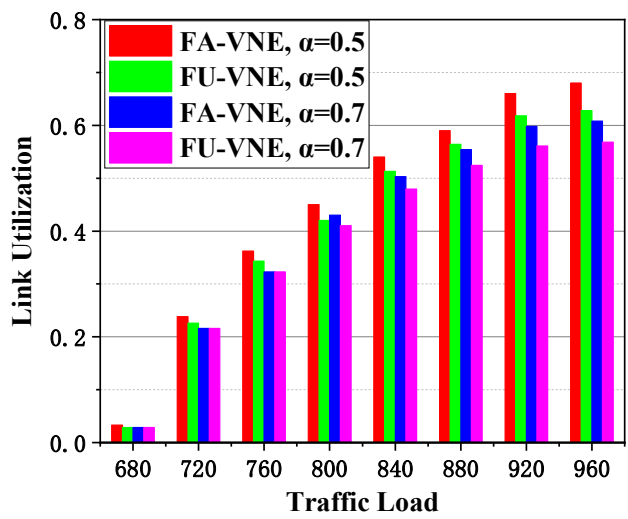

(a)

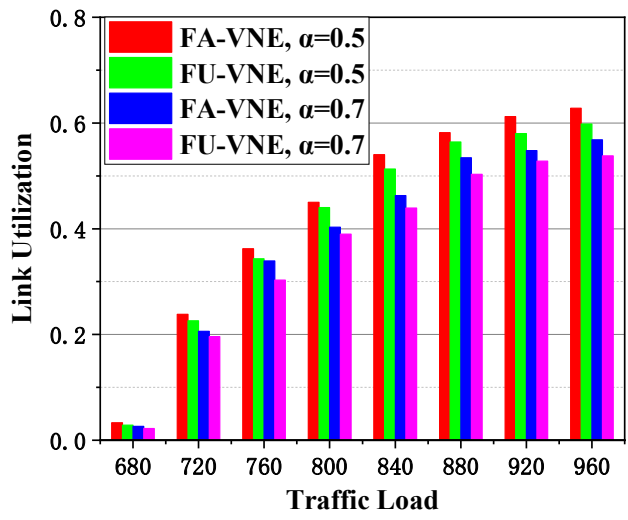

(c)

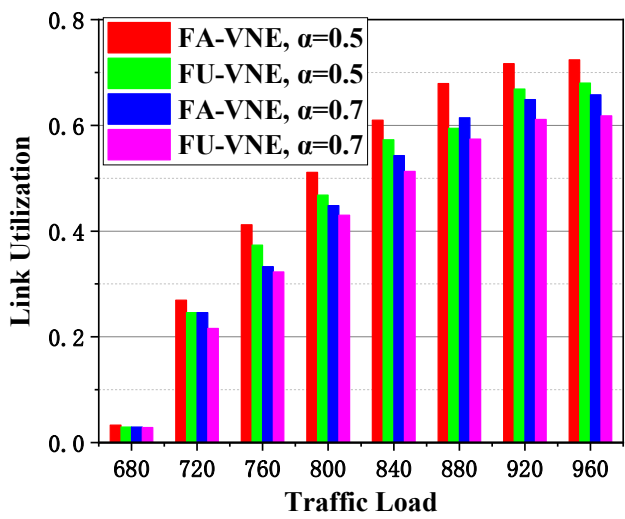

(b)

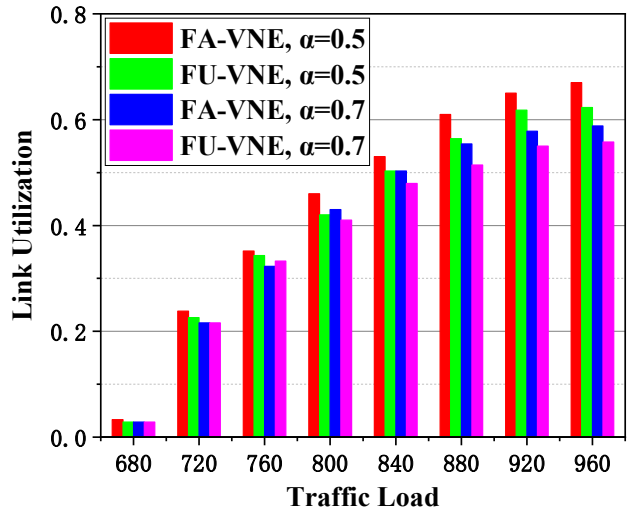

(d)

Figure 5. Simulation and results: (a) LUR under NS-A; (b) LUR under NS-B; (c) LUR under NS-C; and (d) LUR under NS-D.

\section{Conclusions}

Brown-field migration from a fixed-grid to a flexible-grid network will be a long process. In order to make better use of management means to pass this period smoothly; we explore how the problem of VNE can be solved when considering mixed-fixed/flexiblegrid optical infrastructure in this paper. The problem of VON provisioning over co-existing fixed/flexible grid optical networks is introduced firstly. A flexible-grid aware virtual network embedding algorithm is proposed and evaluated under four different network scenarios. Simulation results show that considering a flexible-grid aware approach can largely reduce the blocking probabilities, and a flexible-grid aware approach can lead to significant savings in spectrum resources compared to the cases when no flexible-grid network configuration is taken into consideration. Also, higher probability of virtual link generation can lead to lower blocking probability. In addition, the VNE problem over coexisting fixed/flexible grid optical networks under a static scenario is also very important, and our future work will focus on the ILP model and lower/upper bound analysis of corresponding solutions.

Author Contributions: Conceptualization, X.Y., Y.Z. and J.Z.; methodology, X.Y. and Y.Z.; software, L.L.; validation, L.L. and X.Y.; writing-original draft preparation, X.Y.; writing-review and editing, L.L., Y.Z., F.W., A.N., X.L. and J.Z.; supervision, J.Z. All authors have read and agreed to the published version of the manuscript.

Funding: This work was supported by funding of the science and technology research and development project of Yinchuan (2021BDE13001).

Data Availability Statement: The data presented in this study are available on request from the corresponding author. The data are not publicly available due to privacy.

Conflicts of Interest: The authors declare no conflict of interest. 


\section{References}

1. Zhang, Q.; Xie, W.; She, Q.; Wang, X.; Palacharla, P.; Sekiya, M. RWA f or network virtualization in optical WDM networks. In Proceedings of the 2013 Optical Fiber Communication Conference and Exposition and the National Fiber Optic Engineers Conference (OFC/NFOEC), Anaheim, CA, USA, 17-21 March 2013.

2. Shakya, S.; Pradhan, N.; Cao, X.; Ye, Z.; Qiao, C. Virtual network embedding and reconfiguration in elastic optical networks. In Proceedings of the 2014 IEEE Global Communications Conference, Austin, TX, USA, 8-12 December 2014.

3. Chaudhari, S.; Mani, R.S.; Raundale, P. SDN network virtualization survey. In Proceedings of the 2016 International Conference on Wireless Communications, Signal Processing and Networking (WiSPNET), Chennai, India, 23-25 March 2016.

4. Belbekkouche, A.; Hasan, M.; Karmouch, A. Resource discovery and allocation in network virtualization. IEEE Commun. Surv. Tutor. 2012, 14, 1114-1128. [CrossRef]

5. Chowdhury, N.M.K.; Boutaba, R. A survey of network virtualization. Comput. Netw. 2010, 54, 862-876. [CrossRef]

6. Hwang, J.; Ramakrishnan, K.; Wood, T. NetVM: High performance and flexible networking using virtualization on commodity platforms. IEEE Trans. Netw. Serv. Manag. 2015, 12, 34-47. [CrossRef]

7. Khan, A.; Zugenmaier, A.; Jurca, D.; Kellerer, W. Network virtualization: A hypervisor for the Internet? IEEE Commun. Mag. 2012, 50, 136-143. [CrossRef]

8. Blenk, A.; Basta, A.; Reisslein, M.; Kellerer, W. Survey on Network Virtualization Hypervisors for Software Defined Networking. IEEE Commun. Surv. Tutor. 2016, 18, 655-685. [CrossRef]

9. Barakabitze, A.A.; Ahmad, A.; Mijumbi, R.; Hines, A. 5G network slicing using SDN and NFV: A survey of taxonomy, architectures and future challenges. Comput. Netw. 2020, 167, 106984. [CrossRef]

10. Koponen, T.; Amidon, K.; Balland, P.; Casado, M.; Chanda, A.; Fulton, B.; Ganichev, I.; Gross, J.; Ingram, P.; Jackson, E.; et al. Network virtualization in multi-tenant datacenters. In Proceedings of the 11th USENIX Symposium on Networked Systems Design and Implementation (NSDI 14), Seattle, WA, USA, 2-4 April 2014.

11. Fischer, A.; Botero, J.F.; Beck, M.T.; De Meer, H.; Hesselbach, X. Hesselbach. Virtual network embedding: A survey. IEEE Commun. Surv. Tutor. 2013, 15, 1888-1906. [CrossRef]

12. Dávalos, E.J.; Barán, B. A Survey on Algorithmic Aspects of Virtual Optical Network Embedding for Cloud Networks. IEEE Access 2018, 6, 20893-20906. [CrossRef]

13. Gerstel, O.; Jinno, M.; Lord, A.; Yoo, S.J.B. Elastic optical networking: A new dawn for the optical layer? IEEE Commun. Mag. 2012, 50, s12-s20. [CrossRef]

14. Peng, S.; Nejabati, R.; Simeonidou, D. Impairment-Aware Optical Network Virtualization in Single-Line-Rate and Mixed-Line-Rate WDM Networks. J. Opt. Commun. Netw. 2013, 5, 283-293. [CrossRef]

15. Shahriar, N.; Taeb, S.; Chowdhury, S.R.; Tornatore, M.; Boutaba, R.; Mitra, J.; Hemmati, M. Achieving a Fully-Flexible Virtual Network Embedding in Elastic Optical Networks. In Proceedings of the IEEE INFOCOM-IEEE Conference on Computer Communications, Paris, France, 29 April-2 May 2019.

16. Jinno, M.; Takara, H.; Kozicki, B.; Tsukishima, Y.; Sone, Y.; Matsuoka, S. Spectrum-efficient and scalable elastic optical path network: Architecture, benefits, and enabling technologies. IEEE Commun. Mag. 2009, 47, 66-73. [CrossRef]

17. Zhu, M.; Sun, Q.; Zhang, S.; Gao, P.; Chen, B.; Gu, J. Energy-Aware Virtual Optical Network Embedding in Sliceable-TransponderEnabled Elastic Optical Networks. IEEE Access 2019, 7, 41897-41912. [CrossRef]

18. Ding, S.; Bose, S.; Shen, G. Spectrum trading between virtual optical networks with time-varying traffic in an elastic optical network. J. Opt. Commun. Netw. 2020, 12, 24-37. [CrossRef]

19. Ding, S.; Shen, G.; Pan, K.X.; Bose, S.K.; Zhang, Q.; Mukherjee, B. Blockchain-Assisted Spectrum Trading Between Elastic Virtual Optical Networks. IEEE Netw. 2020, 34, 205-211. [CrossRef]

20. Patel, A.; Ji, P.; Huang, Y.; Wang, T. Distance-Adaptive Virtual Network Embedding in Software-Defined Optical Networks. In Proceedings of the OptoElectronics and Communications Conference Held Jointly with 2013 International Conference on Photonics in Switching, Kyoto, Japan, 30 June-4 July 2013.

21. Lin, R.; Luo, S.; Zhou, J.; Wang, S.; Cai, A.; Zhong, W.; Zukerman, M. Virtual Network Embedding with Adaptive Modulation in Flexi-Grid Networks. J. Lightwave Technol. 2018, 36, 3551-3563. [CrossRef]

22. Zhu, M.; Zhang, S.; Sun, Q.; Li, G.; Chen, B.; Gu, J. Fragmentation-Aware VONE in Elastic Optical Networks. J. Opt. Commun. Netw. 2018, 10, 809-822. [CrossRef]

23. Lin, R.; Luo, S.; Wang, H.; Wang, S. Energy-aware virtual network embedding in flexi-grid networks. Opt. Express 2017, 25, 29699-29713. [CrossRef]

24. Lin, R.; Luo, S.; Zhou, J.; Wang, S.; Chen, B.; Zhang, X.; Cai, A.; Zhong, W.; Zukerman, M. Column generation algorithms for virtual network embedding in flexi-grid optical networks. Opt. Express 2018, 26, 10898-10913. [CrossRef] [PubMed]

25. Yu, X.; Tornatore, M.; Xia, M.; Wang, J.; Zhang, J.; Zhao, Y.; Zhang, J.; Mukherjee, B. Migration from fixed grid to flexible grid in optical networks. IEEE Commun. Mag. 2015, 53, 2. [CrossRef]

26. Mayoral, A.; López, V.; de Dios, O.G.; Fernández-Palacios, J. Migration Steps Toward Flexi-Grid Networks. J. Opt. Commun. Netw. 2014, 6, 988-996. [CrossRef]

27. Yu, X.; Zhao, Y.; Zhang, J.; Mukherjee, B.; Zhang, J.; Wang, X. Static Routing and Spectrum Assignment in Co-existing Fixed/Flex Grid Optical Networks. In Proceedings of the Optical Fiber Communication Conference, OSA Technical Digest (online), San Francisco, CA, USA, 9-13 March 2014. 
28. Yu, X.; Tornatore, M.; Zhao, Y.; Zhang, J.; Wang, X.; Zhang, S.; Wang, R.; Wang, J.; Zhang, J.; Mukherjee, B. When and how should the optical network be upgraded to flex grid? In Proceedings of the European Conference on Optical Communication (ECOC), Cannes, France, 21-25 September 2014.

29. Rofoee, B.; Zervas, G.; Yan, Y.; Amaya, N.; Simeonidou, D. Flexible and Adaptive Optical Metro Networking on Fixed/Flex Grid Exploiting Hybrid Time/Frequency for Shared Resource Allocation. In Proceedings of the European Conference and Exhibition on Optical Communication, OSA Technical Digest (Online), Amsterdam, The Netherlands, 16-20 September 2012.

30. Ahmed, T.; Rahman, S.; Ferdousi, S.; Tornatore, M.; Mitra, A.; Chatterjee, B.C.; Mukherjee, B. Dynamic routing, spectrum, and modulation-format allocation in mixed-grid optical networks. J. Opt. Commun. Netw. 2020, 12, 79-88. [CrossRef]

31. Palkopoulou, E.; Angelou, M.; Klonidis, D.; Christodoulopoulos, K.; Klekamp, A.; Buchali, F.; Varvarigos, E.; Tomkos, I. Quantifying Spectrum, Cost, and Energy Efficiency in Fixed-Grid and Flex-Grid Networks [Invited]. J. Opt. Commun. Netw. 2012, 4, B42-B51. [CrossRef]

32. Papanikolaou, P.; Soumplis, P.; Manousakis, K.; Papadimitriou, G.; Ellinas, G.; Christodoulopoulos, K.; Varvarigos, E. Minimizing Energy and Cost in Fixed-Grid and Flex-Grid Networks. J. Opt. Commun. Netw. 2015, 7, 337-351. [CrossRef]

33. Zong, Y.; Ou, Y.; Hammad, A.; Kondepu, K.; Nejabati, R.; Simeonidou, D.; Liu, Y.; Guo, L. Location-Aware Energy Efficient Virtual Network Embedding in Software-Defined Optical Data Center Networks. J. Opt. Commun. Netw. 2018, 10, B58-B70. [CrossRef]

34. Zhu, Q.; Yu, X.; Zhao, Y.; Nag, A.; Zhang, J. Auxiliary-Graph-Based Energy-Efficient Traffic Grooming in IP-Over-Fixed/Flex-Grid Optical Networks. J. Lightwave Technol. 2021, 39, 3011-3024. [CrossRef]

35. Yu, X.; Lu, L.; Zhao, Y.; Zhang, J. VON Provisioning Over Co-Existing Fixed/Flexible Grid Optical Networks. In Proceedings of the Opto-Electronics and Communications Conference (OECC), Hongkong, China, 3-7 July 2021. 